Ciência Florestal, Santa Maria, v. 25, n. 4, p. 1001-1014, out.-dez., 2015

ISSN 0103-9954

\title{
UMIDADE DE EQUILÍBRIO DE PAINÉIS OSB EM FUNÇÃO DA UMIDADE RELATIVA E DA TEMPERATURA AMBIENTE
}

\author{
EQUILIBRIUM MOISTURE CONTENT OF OSB PANELS AS A FUNCTION OF RELATIVE \\ HUMIDITY AND AMBIENT TEMPERATURE
}

\author{
Rafael Farinassi Mendes ${ }^{1}$ Lourival Marin Mendes ${ }^{2}$ Thiago de Paula Protásio ${ }^{3}$ \\ Stefânia Lima Oliveira ${ }^{3}$ Amélia Guimarães Carvalho $^{4}$ Camila Lais Farrapo $^{5}$
}

\begin{abstract}
RESUMO
O trabalho teve como objetivo obter modelos estatísticos para a estimativa da umidade de equilíbrio de painéis OSB em função da temperatura e da umidade relativa do ar, assim como também avaliar o efeito de algumas variáveis de produção sobre a umidade de equilíbrio dos painéis. O delineamento experimental se constituiu de seis condições de processamento, três temperaturas do ar e seis umidades relativas do ar. Nas condições de processamento, foram avaliadas três diferentes espessuras das partículas strand $(0,4 ; 0,7$ e $1,0 \mathrm{~mm})$, duas densidades aparentes do painel $\left(0,65\right.$ e $\left.0,90 \mathrm{~g} / \mathrm{cm}^{3}\right)$ e também três níveis de pressão na prensagem dos painéis $\left(40,60\right.$ e $\left.80 \mathrm{kgf} / \mathrm{cm}^{2}\right)$. Para cada tratamento foram produzidos quatro painéis com a madeira de Pinus taeda e 6\% de adesivo fenol-formaldeído. Na avaliação do experimento foi considerado um delineamento inteiramente casualizado disposto em esquema fatorial triplo $6 \times 3 \times 6$, ou seja, 6 variáveis de produção (condições de processamento), 3 temperaturas do ar $\left(30,40\right.$ e $\left.50^{\circ} \mathrm{C}\right)$ e 6 umidades relativas $(40,50,60,70,80,90 \%)$. As médias foram comparadas estatisticamente pelo Teste Scott-Knott em nível de 5\% de significância. A modelagem da umidade de equilíbrio dos painéis OSB foi realizada mediante o ajuste de modelos polinomiais múltiplos para cada tratamento. Com base nas medidas de precisão e nos resultados obtidos pode-se concluir que: 1) recomenda-se a utilização do modelo $\mathrm{UEQ}=\beta_{0}+\beta_{1} \mathrm{UR}+\beta_{2} \mathrm{UR}^{2}+\beta_{3} \mathrm{UR}^{3}+\beta_{4}$ Temp $+\varepsilon$ para a estimativa indireta da umidade de equilíbrio dos painéis OSB; 2) A temperatura apresenta influência linear na umidade de equilíbrio dos painéis, enquanto que a umidade relativa do ar apresenta comportamento polinomial de terceira ordem, sendo que a umidade relativa do ar influencia de forma mais pronunciada a umidade de equilíbrio dos painéis OSB do que a temperatura ambiente; 3) Quanto ao efeito das variáveis de produção, a pressão de prensagem de $80 \mathrm{kgf} /$ $\mathrm{cm}^{2}$ e o aumento da espessura das partículas strand para 1,0 $\mathrm{mm}$ de espessura promoveu tendência de reduções nos valores médios de umidade de equilíbrio dos painéis OSB. Já o aumento da densidade do painel promoveu uma tendência de aumento da umidade de equilíbrio dos painéis OSB; e 4) O uso de modelos polinomiais múltiplos permite que sejam produzidas curvas de nível para a obtenção dos valores de umidade de equilíbrio dos painéis OSB em função da umidade relativa e da temperatura do local onde o painel esta exposto, se destacando pela sua praticidade de utilização.
\end{abstract}

Palavras-chave: Pinus taeda; chapas aglomeradas estruturais OSB; modelagem; umidade de equilíbrio.

1 Engenheiro Florestal, Dr., Professor da Engenharia de Materiais, Universidade Federal de Lavras, Caixa Postal 3037, CEP 37200-000, Lavras (MG), Brasil. rafaelfarinassi@gmail.com

2 Engenheiro Florestal, Dr., Professor do Departamento de Engenharia Florestal, Universidade Federal de Lavras, Caixa Postal 3037, CEP 37200-000, Lavras (MG), Brasil. lourival@dcf.ufla.br

3 Engenheiro Florestal, Doutorandos em Ciência e Tecnologia da Madeira, Universidade Federal de Lavras, Caixa Postal 3037, CEP 37200-000, Lavras (MG), Brasil. depaulaprotassio@gmail.com, stefaniaoliveira@yahoo.com. br

4 Engenheiro Florestal, MSc., Doutoranda em Ciências Florestais, Universidade Federal de Viçosa, CEP36570-000, Viçosa (MG), Brasil. ameliagcarvalho@gmail.com

5 Engenheira Florestal, Doutorando em Engenharia de Biomateriais, Universidade Federal de Lavras, Caixa Postal 3037, CEP 37200-000, Lavras (MG), Brasil. camilafarrapo@hotmail.com

Recebido para publicação em 16/11/2011 e aceito em 31/03/2014

Ci. Fl., v. 25, n. 4, out.-dez., 2015 


\begin{abstract}
The study aimed to obtain statistical models to estimate the equilibrium moisture content of OSB panels as a function of temperature and relative humidity of air, as well as evaluate the effect of some production variables on the equilibrium moisture content of the panels. The experimental design consisted of six processing conditions, three air temperature and six relative humidity of air. In the processing conditions, were evaluated three different thicknesses of the strand particles $(0.4,0.7$ and $1.0 \mathrm{~mm})$, two apparent densities of panels $\left(0.65\right.$ and $\left.0.90 \mathrm{~g} / \mathrm{cm}^{3}\right)$ and three levels of pressure in the pressing of the panels $(40,60$ and 80 $\left.\mathrm{kgf} / \mathrm{cm}^{2}\right)$. For each treatment four panels were produced with the wood of Pinus taeda and $6 \%$ of phenol formaldehyde adhesive. In the evaluation of the experiment was considered a completely randomized design arranged in a factorial triple $6 \times 6 \times 3$, in order words, six production variables (processing conditions), three air temperatures $\left(30,40\right.$ and $\left.50^{\circ} \mathrm{C}\right)$ and 6 relative humidity $(40,50,60,70,80,90 \%)$. The means were compared statistically by Scott-Knott test at the 5\% level of significance. The modeling the equilibrium moisture content of OSB panels was performed with fit the multiple polynomial models for each treatment. Based on measurements of accuracy and the results can be concluded that: 1) it is recommended to use the model UEQ $=\beta_{0}+\beta_{1} \mathrm{UR}+\beta_{2} \mathrm{UR}^{2}+\beta_{3} \mathrm{UR}^{3}+\beta_{4}$ Temp $+\varepsilon$ for indirect estimation of equilibrium moisture content of OSB panels 2) The temperature shows linear influence on the equilibrium moisture content of the panels, while the relative humidity of air shows behaving of third order polynomial, and the relative humidity of air affects more pronouncedly the equilibrium moisture content of OSB panels than the ambient temperature; 3) In respect of the effect of production variables, the pressing of pressure of $80 \mathrm{kgf} / \mathrm{cm}^{2}$ and the increased the thickness of the strand particles to $1.0 \mathrm{~mm}$ thick promoted trend of reductions in average of the equilibrium moisture content of OSB panels. But the increased density of the panel promoted the trend of increasing of equilibrium moisture content of OSB panels; and 4) The use of multiple polynomial models allows that are produced contours to obtain the values of equilibrium moisture content of OSB as a function of relative humidity and temperature of the place where the panel is exposed, standing out for its convenience of use.
\end{abstract}

Keywords: Pinus taeda; structural particleboard (OSB); modeling; equilibrium moisture.

\section{INTRODUÇÃO}

Os painéis OSB (Oriented Strand Board) são produzidos a partir de partículas de madeira do tipo strand, sendo constituídos por três camadas perpendiculares entre si e colados com adesivo sintético. Sua utilização abrange uma ampla gama de produtos, dentre as quais a produção de paredes, pisos, móveis, forros, componentes de vigas e embalagens.

Tais painéis por serem constituídos de madeira, um material higroscópico, quando em contato com o ar, absorvem ou perdem umidade no estado líquido ou de vapor até atingir um equilíbrio (MARTINS et al., 2003). De acordo com Simpson e TenWolde (1999), a umidade de equilíbrio da madeira é uma função da umidade relativa e da temperatura do ar circundante. Sendo que, de acordo com Siau (1995), a umidade relativa do ar é o principal fator na umidade de equilíbrio da madeira.

No entanto, de forma geral, os produtos reconstituídos de madeira apresentam uma higroscopicidade diferente em relação à madeira maciça. Este fato é devido à redução em lâminas, partículas e a posterior incorporação de diferentes teores e tipos de adesivos, parafinas e outros produtos (SILVA et al., 2006).

Outro aspecto que contribui para a redução da higroscopicidade destes produtos é a utilização de altas temperaturas e pressão na consolidação final do painel (WU, 1999), as quais podem causar degradação de alguns constituintes químicos, principalmente as hemiceluloses, promovendo a diminuição da umidade de equilíbrio dos painéis (MENDES et al., 2012a).

Mendes et al. (2006), estudando o efeito das variáveis de processo sobre a umidade de equilíbrio de painéis particulados, concluíram que a umidade de equilíbrio foi afetada pela temperatura de prensagem e pela interação entre a temperatura e o tempo de prensagem. Enquanto que Silva et al. (2006) avaliando a umidade de equilíbrio de painéis OSB observaram que o teor de resina, a temperatura e o tempo de prensagem afetam de 
forma significativa a retenção de água pelos painéis.

No entanto, outros fatores podem vir a afetar a umidade de equilíbrio dos painéis, dentre os quais a densidade, o tipo de painel e a pressão de prensagem, as quais contribuem para a diferença de distribuição e ligação das partículas dos painéis, promovendo diferenças quanto ao ganho de umidade (SILVA et al., 2005; MENDES e ARCE, 2003), assim como também a pressão de prensagem, a espessura das partículas, extrativos da madeira, entre outros (IWAKIRI, 2005).

As variações referentes à perda ou ganho de umidade têm grande importância para o uso adequado de cada painel, já que deformações referentes a essa reação ao contato com a umidade são indesejáveis e apresentam relação direta com as suas demais propriedades. Podendo vir a afetar, de forma bastante significativa, a resistência mecânica e a estabilidade dimensional dos diferentes produtos de madeira (SILVA et al., 2005; BAHAROGLU et al., 2012).

A modelagem da umidade de equilíbrio de painéis de madeira pode ser muito útil para a determinação das corretas condições de uso desses produtos e na diminuição dos custos e do tempo relacionados a essa análise. O uso de modelos lineares múltiplos que relacionam a umidade de equilíbrio dos painéis de madeira em função da umidade relativa e da temperatura do ar permite a construção de curvas de nível e a determinação das melhores condições ambientais visando à maior durabilidade dos painéis. Segundo Werkema e Aguiar (2006), a análise de regressão pode ser utilizada com o objetivo de controlar, descrever, predizer ou estimar a variável de interesse em faixas de valores pré-fixados, reforçando a aplicabilidade do ajuste de modelos para o estudo da umidade de equilíbrio de painéis de madeira reconstituída.

$\mathrm{Na}$ literatura existem equações como a de Nelson (1983) ajustada por Wu (1999) que estimam a umidade de equilíbrio de produtos à base de madeira. Contudo, essas equações além de mais complexas tendem a subestimar a umidade de equilíbrio dos painéis de madeira (SILVA et al., 2005). Nesse contexto, o presente trabalho teve como objetivo obter modelos estatísticos para a estimativa da umidade de equilíbrio de painéis OSB em função apenas da temperatura e da umidade relativa do ar, assim como também avaliar o efeito de algumas variáveis de produção sobre a umidade de equilíbrio dos painéis.

\section{MATERIAL E MÉTODOS}

\section{Matéria-prima}

Os painéis OSB foram confeccionados com a madeira de Pinus taeda L., com idade de 30 anos, densidade básica média de $0,405 \mathrm{~g} / \mathrm{cm}^{3}$ e procedentes de um plantio homogêneo localizado na Estação Experimental do Canguiri, da Universidade Federal do Paraná (UFPR), Município de Pinhais - PR.

\section{Manufatura dos painéis}

Para a produção dos painéis foram consideradas seis condições de processamento, conforme descrito na Tabela 1. Sendo avaliadas diferentes espessuras das partículas strand $(0,4 ; 0,7$ e $1,0 \mathrm{~mm})$, duas densidades aparentes dos painéis $\left(0,65\right.$ e $\left.0,90 \mathrm{~g} / \mathrm{cm}^{3}\right)$ e também três níveis de pressão na prensagem dos painéis $\left(40,60\right.$ e $\left.80 \mathrm{kgf} / \mathrm{cm}^{2}\right)$. Para cada tratamento foram produzidos quatro painéis.

As partículas do tipo strand foram obtidas

TABELA 1: Variáveis utilizadas para a produção dos painéis OSB.

TABLE 1: Variable used for the production of OSB panels.

\begin{tabular}{cccc}
\hline Condições de processamento & $\begin{array}{c}\text { Pressão de prensagem } \\
\left(\mathrm{kgf} / \mathrm{cm}^{2}\right)\end{array}$ & $\begin{array}{c}\text { Densidade do painel } \\
\left(\mathrm{g} / \mathrm{cm}^{3}\right)\end{array}$ & $\begin{array}{c}\text { Espessura das } \\
\text { partículas }(\mathrm{mm})\end{array}$ \\
\hline 1 & 40 & 0,65 & 0,7 \\
2 & 40 & 0,65 & 0,4 \\
3 & 40 & 0,65 & 1,0 \\
4 & 40 & 0,90 & 0,7 \\
5 & 60 & 0,65 & 0,7 \\
6 & 80 & 0,65 & 0,7 \\
\hline
\end{tabular}


mediante o processamento dos blocos de madeira em um picador de discos, sendo este ajustado para gerar partículas de $0,4 \mathrm{~mm}, 0,7 \mathrm{~mm}$ e $1 \mathrm{~mm}$ de espessura, dependendo da condição preestabelecida (Tabela 1). O comprimento e a largura das partículas de todos os painéis produzidos foram de $85 \mathrm{~mm}$ e 25 $\mathrm{mm}$, respectivamente.

Após a geração das partículas estas foram peneiradas para a retirada dos finos e levadas para uma estufa com temperatura de $65 \pm 5{ }^{\circ} \mathrm{C}$, até que atingissem umidade final de 3\% (base massa seca das partículas), sendo posteriormente colocadas em uma encoladeira do tipo tambor giratório, na qual se realizou a aplicação de $6 \%$ de sólidos de resina fenol-formaldeído (base massa seca das partículas).

As partículas encoladas foram então distribuídas sobre uma caixa orientadora, conforme proporções em massa de $25 \%, 50 \%$ e $25 \%$ (face/ miolo/face, respectivamente), sendo o miolo disposto de forma perpendicular às faces. O colchão formado foi então submetido a uma pré-prensagem a frio em uma prensa manual na pressão de $4 \mathrm{kgf} /$ $\mathrm{cm}^{2}$, seguido por uma prensagem a quente com um ciclo de prensagem de 8 minutos, temperatura de $180^{\circ} \mathrm{C}$ e pressão de prensagem de $40,60 \mathrm{ou} 80 \mathrm{kgf} /$ $\mathrm{cm}^{2}$, dependendo da condição de processamento preestabelecida (Tabela 1).

\section{Avaliação da umidade de equilíbrio}

Para cada condição de processamento foram retirados 24 corpos de prova, sendo seis para cada painel. As dimensões dos corpos de prova foram de $25 \times 25 \mathrm{~mm}$, conforme metodologia desenvolvida por $\mathrm{Wu}(1999)$.

Os corpos de prova foram então divididos em três grupos de oito amostras, sendo cada grupo levado para uma câmara de climatização regulada com temperatura de $30^{\circ} \mathrm{C}, 40^{\circ} \mathrm{C}$ e $50^{\circ} \mathrm{C}$, na qual se efetuou a variação da umidade relativa do ar $(40,50$, $60,70,80$ e 90\%). Após a estabilização dos corpos de prova, em cada uma das condições de temperatura e umidade relativa, foi efetuada a determinação da massa dos mesmos.

Ao final, todos os corpos de prova foram levados para uma estufa com temperatura de $103 \pm 2^{\circ} \mathrm{C}$, o que permitiu o registro da massa seca de cada corpo de prova e, por consequência, proporcionou a determinação da umidade de equilíbrio das amostras em cada condição de temperatura e umidade.

\section{Análises estatísticas}

$\mathrm{Na}$ avaliação do experimento foi considerado um delineamento inteiramente casualizado disposto em esquema fatorial triplo $6 \times 3 \times 6$, ou seja, 6 condições de processamento, 3 temperaturas do ar e 6 umidades relativas. As médias foram comparadas estatisticamente pelo Teste Scott-Knott em nível de 5\% de significância.

A modelagem da umidade de equilíbrio dos painéis OSB foi realizada mediante o ajuste de modelos polinomiais múltiplos para cada tratamento considerado, conforme apresentado na Tabela 2.

Foram realizadas análises de variância e verificada a significância dos coeficientes dos modelos por meio do teste $\mathrm{t}$ a $5 \%$. Os melhores modelos foram selecionados com base no coeficiente de determinação ajustado $\left(\mathrm{R}_{\text {ajust }}^{2}\right)$, no erro padrão da estimativa $\left(\mathrm{S}_{\mathrm{xy}}\right)$ e no erro padrão da estimativa em porcentagem $\left(\mathrm{S}_{\mathrm{xy} \%}\right)$. Para melhorar a qualidade dos ajustes foram removidos dos modelos os outliers ou observações extremas.

Para o melhor modelo selecionado foram avaliadas as curvas de nível (ou gráficos

TABELA 2: Modelos estatísticos propostos.

TABLE 2: Proposed statistical models.

\begin{tabular}{cc}
\hline N. do modelo & Modelos estatísticos \\
\hline 1 & $\mathrm{UEQ}=\beta_{0}+\beta_{1} \mathrm{UR}+\beta_{2} \mathrm{UR}^{2}+\beta_{3} \mathrm{UR}^{3}+\beta_{4}$ Temp $+\varepsilon$ \\
2 & $\mathrm{UEQ}=\beta_{0}+\beta_{1} \mathrm{UR}+\beta_{2} \mathrm{UR}^{2}+\beta_{3} \mathrm{UR}^{3}+\beta_{4}$ Temp $+\beta_{5} \mathrm{Temp}^{2}+\varepsilon$ \\
3 & $\mathrm{UEQ}=\beta_{0}+\beta_{1} \mathrm{UR}+\beta_{2} \mathrm{UR}^{2}+\beta_{3}$ Temp $+\beta_{4}$ Temp $^{2}+\varepsilon$ \\
4 & $\mathrm{UEQ}=\beta_{0}+\beta_{1} \mathrm{UR}+\beta_{2} \mathrm{UR}^{2}+\beta_{3}$ Temp $+\varepsilon$ \\
\hline
\end{tabular}

Em que: UEQ = umidade de equilíbrio; UR = umidade relativa do ar; Temp = temperatura média do ar; $\beta_{0}, \beta_{1}, \beta_{2}, \beta_{3}$, $\beta_{4}, \beta_{5}=$ coeficientes dos modelos $\varepsilon=$ resíduo do modelo. 
de contornos), visando explicar a influência da umidade de equilíbrio e da temperatura na umidade de equilíbrio dos painéis OSB. Além disso, a análise das curvas de nível permite encontrar as melhores condições de temperatura e umidade relativa para a utilização dos painéis.

\section{RESULTADOS E DISCUSSÃO}

$\mathrm{Na}$ Tabela 3 encontra-se um resumo da análise de variância efetuada. Observa-se interação significativa entre as condições de processamento, temperaturas e umidades relativas do ar, ou seja, há dependência entre os fatores considerados. Dessa forma, optou-se pelo desdobramento de cada condição de processamento dentro de cada nível de temperatura e umidade relativa do ar.
$\mathrm{Na}$ Figura 1 encontram-se os valores médios para a umidade de equilíbrio considerando os níveis dos desdobramentos que não apresentaram efeito significativo para as diferentes condições de processamento. Observa-se que para a temperatura de $30^{\circ} \mathrm{C}$ ocorreu variações consideráveis na umidade de equilíbrio dos painéis em função da modificação apenas da umidade relativa do ar. Esse resultado demonstra a grande importância dessa variável na determinação de modelos para predição da umidade de equilíbrio dos painéis. Além de evidenciar a relevância da determinação da umidade de equilíbrio dos painéis, essa variável é fundamental na escolha dos locais mais adequados para a utilização dos painéis, corroborando com Siau (1995).

O local pode ocasionar grande variação na sua umidade de equilíbrio, a qual pode influenciar

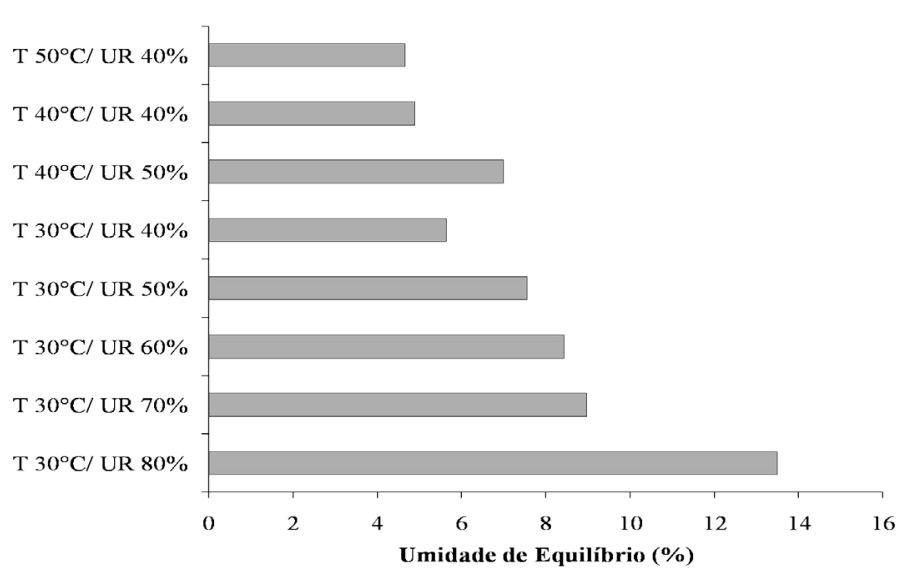

FIGURA 1: Valores médios para a umidade de equilíbrio considerando os níveis do desdobramento que não apresentaram efeito significativo para as condições de processamento

FIGURE 1: Mean values for the equilibrium moisture content considering the levels of development that had no significant effect for the processing conditions.

TABELA 3: Resumo da análise de variância.

TABLE 3: Analysis of variance summary.

\begin{tabular}{lcc}
\hline Fator de Variação & Graus de Liberdade & Quadrado Médio \\
\hline Condição de processamento & 5 & $7,2282^{* *}$ \\
Temperatura & 2 & $41,4099^{* *}$ \\
Umidade relativa & 5 & $4480,2130^{* *}$ \\
Condição de processamento x Temperatura & 10 & $0,2485^{\text {ns }}$ \\
Condição de processamento x Umidade relativa & 25 & $0,4516^{* *}$ \\
Temperatura*Umidade relativa & 10 & $51,3771^{* *}$ \\
Condição de processamento x Temperatura x Umidade relativa & 50 & $0,3388^{*}$ \\
Erro & 751 & 0,2270 \\
Coeficiente de Variação $=4,52 \%$ & & \\
\hline
\end{tabular}

Em que: $*$ = significativo a $5 \%$ pelo Teste $\mathrm{F} ;{ }^{* *}=$ significativo a $1 \%$ pelo Teste $\mathrm{F} ; \mathrm{ns}=$ não significativo a $5 \%$ pelo Teste F. 
de forma bastante acentuada suas propriedades mecânicas, o que é algo a se levar em consideração, já que os painéis OSB são destinados a aplicações estruturais (BAHAROGLU et al., 2012).

\section{Avaliação das condições de processamento}

Na Tabela 4 encontram-se os valores médios e teste de comparação múltipla considerando-se os níveis do desdobramento que apresentaram efeito significativo para as condições de processamento.

De forma geral, observou-se tendência de diminuição da umidade de equilíbrio dos painéis OSB à medida que se aumentou a pressão de prensagem dos painéis, sendo que a pressão de $80 \mathrm{kgf} / \mathrm{cm}^{2}$ proporcionou diminuições dos valores médios em relação aos painéis tomados como padrões (Condição de processamento 1), se diferenciando estatisticamente desses na maioria dos casos.

Para a espessura das partículas strand se observou, apenas em algumas condições de umidade relativa e temperatura, a diminuição significativa da umidade de equilíbrio dos painéis OSB produzidos com as partículas strand de $1,0 \mathrm{~mm}$ de espessura. Diferentemente do obtido por Haselein et al. (2002), os quais avaliando diferentes dimensões de partículas sobre as propriedades de painéis produzidos com adesivo a base de tanino-formaldeído, não observaram efeito significativo entre as espessuras de 0,5 e 1,0 $\mathrm{mm}$ das partículas sobre a umidade de equilíbrio dos painéis. No caso do presente estudo, tal fato pode estar associado à quantidade de partículas necessárias para compor o colchão do painel, visto que o aumento da espessura das partículas causa a diminuição do número de partículas necessário para uma mesma densidade do painel e, consequentemente, reduz a área superficial apta para realizar ligações com a umidade do ar.

Quanto à densidade do painel, observase que os painéis produzidos com densidade de $0,90 \mathrm{~g} / \mathrm{cm}^{3}$ (Condição de processamento 4) obtiveram os maiores valores médios de umidade de equilíbrio dos painéis OSB em relação à condição de processamento padrão (Condição de processamento 1), apresentando-se com diferença

TABELA 4: Valores médios e teste de comparação múltipla considerando-se os níveis do desdobramento que apresentaram efeito significativo para as condições de processamento.

TABLE 4: Mean values and multiple comparison test considering the levels of development that showed significant effect for the processing conditions.

\begin{tabular}{|c|c|c|c|c|c|c|c|c|c|}
\hline \multicolumn{2}{|c|}{$\mathrm{T} 30^{\circ} \mathrm{C} / \mathrm{UR} 90 \%$} & \multicolumn{2}{|c|}{$\mathrm{T} 40^{\circ} \mathrm{C} / \mathrm{UR} 60 \%$} & \multicolumn{2}{|c|}{$\mathrm{T} 40^{\circ} \mathrm{C} / \mathrm{UR} 70 \%$} & \multicolumn{2}{|c|}{$\mathrm{T} 40^{\circ} \mathrm{C} / \mathrm{UR} 80 \%$} & \multicolumn{2}{|c|}{$\mathrm{T} 40^{\circ} \mathrm{C} / \mathrm{UR} 90 \%$} \\
\hline UEQ & C. $p$ & UEQ & C. $p$ & UEQ & C. $p$ & UEQ & C. $p$ & UEQ & C. $p$ \\
\hline $21,11 \mathrm{~b}$ & 1 & $8,29 \mathrm{~b}$ & 1 & 8,79 a & 1 & $13,18 \mathrm{~b}$ & 1 & $22,23 \mathrm{~b}$ & 1 \\
\hline $21,38 \mathrm{~b}$ & 2 & $8,48 \mathrm{~b}$ & 2 & 8,78 a & 2 & $12,93 \mathrm{a}$ & 2 & $22,75 \mathrm{~b}$ & 2 \\
\hline 20,46 a & 3 & $7,79 \mathrm{a}$ & 3 & $8,32 \mathrm{a}$ & 3 & $12,70 \mathrm{a}$ & 3 & $21,97 \mathrm{~b}$ & 3 \\
\hline 20,76 a & 4 & $8,36 \mathrm{~b}$ & 4 & $9,11 \mathrm{~b}$ & 4 & $13,36 \mathrm{~b}$ & 4 & $22,86 \mathrm{c}$ & 4 \\
\hline $20,47 \mathrm{a}$ & 5 & $7,79 \mathrm{a}$ & 5 & $9,11 \mathrm{~b}$ & 5 & $13,36 \mathrm{~b}$ & 5 & $22,86 \mathrm{c}$ & 5 \\
\hline $20,44 \mathrm{a}$ & 6 & $7,94 \mathrm{a}$ & 6 & 8,67 a & 6 & $12,87 \mathrm{a}$ & 6 & $21,57 \mathrm{a}$ & 6 \\
\hline \multicolumn{2}{|c|}{ T50 ${ }^{\circ} \mathrm{C} / \mathrm{UR} 50 \%$} & \multicolumn{2}{|c|}{$\mathrm{T} 50^{\circ} \mathrm{C} / \mathrm{UR} 60 \%$} & \multicolumn{2}{|c|}{$\mathrm{T} 50^{\circ} \mathrm{C} / \mathrm{UR} 70 \%$} & \multicolumn{2}{|c|}{$\mathrm{T} 50^{\circ} \mathrm{C} / \mathrm{UR} 80 \%$} & \multicolumn{2}{|c|}{$\mathrm{T} 50^{\circ} \mathrm{C} / \mathrm{UR} 90 \%$} \\
\hline UEQ & C. $p$ & UEQ & C. $p$ & UEQ & C. $p$ & UEQ & C. $p$ & UEQ & C. $p$ \\
\hline $6,16 \mathrm{a}$ & 1 & $8,27 \mathrm{~b}$ & 1 & $10,16 \mathrm{~b}$ & 1 & $13,69 \mathrm{~b}$ & 1 & $17,99 \mathrm{a}$ & 1 \\
\hline $6,10 \mathrm{a}$ & 2 & $8,22 \mathrm{~b}$ & 2 & $10,24 \mathrm{~b}$ & 2 & $14,04 \mathrm{~b}$ & 2 & $18,08 \mathrm{a}$ & 2 \\
\hline $5,77 \mathrm{a}$ & 3 & $7,59 \mathrm{a}$ & 3 & $9,91 \mathrm{a}$ & 3 & $13,84 \mathrm{~b}$ & 3 & 17,70 a & 3 \\
\hline $6,69 \mathrm{~b}$ & 4 & $8,17 \mathrm{~b}$ & 4 & $10,42 \mathrm{~b}$ & 4 & $14,20 \mathrm{~b}$ & 4 & $18,83 \mathrm{~b}$ & 4 \\
\hline $6,24 \mathrm{a}$ & 5 & $7,94 \mathrm{a}$ & 5 & $10,42 \mathrm{~b}$ & 5 & $13,32 \mathrm{a}$ & 5 & 17,89 a & 5 \\
\hline $6,11 \mathrm{a}$ & 6 & $7,78 \mathrm{a}$ & 6 & $9,67 \mathrm{a}$ & 6 & $13,03 \mathrm{a}$ & 6 & $17,74 \mathrm{a}$ & 6 \\
\hline
\end{tabular}

Em que: $\mathrm{C} . \mathrm{p}=$ Condições de processamento; UEQ = umidade de equilíbrio (\%); Trat $=$ tratamentos; UR = umidade relativa do ar; $\mathrm{T}=$ temperatura. Médias seguidas pela mesma letra não diferem a $\%$ de significância pelo Teste ScottKnott. 
estatística em alguns casos. Mendes et al. (2012b), avaliando a umidade de equilíbrio de diferentes tipos de painéis de madeira, também observaram aumento da umidade de equilíbrio dos painéis OSB com o aumento da densidade do painel. A explicação do fato se deve a maior quantidade de material higroscópico, ou seja, maior quantidade de madeira, a qual proporciona maior número de ligações do tipo - $\mathrm{OH}$.

\section{Modelagem da umidade de equilíbrio}

Na Tabela 5 estão apresentados os parâmetros e estatísticas referentes ao modelo ajustado 1 para as diferentes condições de processamento.

Observa-se que todos os modelos ajustados foram significativos pelo Teste $\mathrm{F}$, apresentaram todos os coeficientes significativos pelo Teste t, elevado coeficiente de determinação ajustado e baixo erro

TABELA 5: Parâmetros e estatísticas referentes ao modelo ajustado 1 para as diferentes condições de processamento.

TABLE 5: Parameters and statistics relating to model adjusted 1 for the different processing conditions.

\begin{tabular}{|c|c|c|c|c|c|c|c|}
\hline C. de processamento & Coeficientes & tc & $\mathrm{Fc}$ & $\mathrm{R}_{\text {ajust }}^{2}$ & $S_{x y}$ & $\mathrm{~S}_{\mathrm{xy} \%}$ & GLerro \\
\hline & $-4,187.10^{1}$ & $-7,22^{* *}$ & & & & & \\
\hline & $2,539 \cdot 10^{0}$ & $8,84^{* *}$ & & & & & \\
\hline \multirow[t]{5}{*}{1} & $-4,335.10^{-2}$ & $-9,49^{* *}$ & $1090^{* *}$ & 0,9682 & 0,92 & 8,67 & 139 \\
\hline & $2,559 \cdot 10^{-4}$ & $10,95^{* *}$ & & & & & \\
\hline & $-3,910.10^{-2}$ & $-4,16^{* *}$ & & & & & \\
\hline & $-4,457.10^{1}$ & $-6,89^{* *}$ & & & & & \\
\hline & $2,658.10^{0}$ & $8,29^{* *}$ & & & & & \\
\hline \multirow[t]{5}{*}{2} & $-4,526.10^{-2}$ & $-8,87^{* *}$ & $936,9^{* *}$ & 0,9637 & 1,02 & 9,54 & 137 \\
\hline & $2,662.10^{-4}$ & $10,19^{* *}$ & & & & & \\
\hline & $-3,513.10^{-2}$ & $-3,38^{* *}$ & & & & & \\
\hline & $\begin{array}{ll}\beta_{0} & -3,871.10^{1}\end{array}$ & $-6,09^{* *}$ & & & & & \\
\hline & $2,338.10^{0}$ & $7,43^{* *}$ & & & & & \\
\hline \multirow[t]{5}{*}{3} & $-4,010.10^{-2}$ & $-8,01^{* *}$ & $924,6^{* *}$ & 0,9630 & 1,00 & 9,84 & 138 \\
\hline & $2,39410^{-4}$ & $9,35^{* *}$ & & & & & \\
\hline & $-3,108.10^{-2}$ & $-3,00^{* *}$ & & & & & \\
\hline & $-3,886.10^{1}$ & $-6,90^{* *}$ & & & & & \\
\hline & $2,371.10^{0}$ & $8,50^{* *}$ & & & & & \\
\hline \multirow[t]{5}{*}{4} & $-4,079.10^{-2}$ & $-9,19^{* *}$ & $1198^{* *}$ & 0,9710 & 0,89 & 8,25 & 139 \\
\hline & $2,438.10^{-4}$ & $10,74^{* *}$ & & & & & \\
\hline & $-2,515 \cdot 10^{-2}$ & $-2,75^{* *}$ & & & & & \\
\hline & $-3,497.10^{1}$ & $-5,62^{* *}$ & & & & & \\
\hline & $2,185.10^{\circ}$ & $7,10^{* *}$ & & & & & \\
\hline \multirow[t]{5}{*}{5} & $-3,778.10^{-2}$ & $-7,72^{* *}$ & $956^{* *}$ & 0,9639 & 0,99 & 9,34 & 139 \\
\hline & $2,280.10^{-4}$ & $9,10^{* *}$ & & & & & \\
\hline & $-3,463 \cdot 10^{-2}$ & $-3,43^{* *}$ & & & & & \\
\hline & $-3,889.10^{1}$ & $-7,46^{* *}$ & & & & & \\
\hline & $2,384.10^{0}$ & $9,23^{* *}$ & & & & & \\
\hline \multirow[t]{3}{*}{6} & $-4,083 \cdot 10^{-2}$ & $-9,93^{* *}$ & $1297^{* *}$ & 0,9735 & 0,83 & 8,00 & 137 \\
\hline & $2,424 \cdot 10^{-4}$ & $11,51^{* *}$ & & & & & \\
\hline & $-4,175.10^{-2}$ & $-4,95^{* *}$ & & & & & \\
\hline
\end{tabular}

Em que: $\mathrm{C}$. de processamento $=$ condições de processamento; $\mathrm{tc}=$ valor de $\mathrm{t}$ calculado; $\mathrm{Fc}=$ valor de $\mathrm{F}$ calculado; $\mathrm{R}^{2}$ ajus = coeficiente de determinação ajustado; $\mathrm{S}_{\mathrm{xy}}=$ erro padrão da estimativa (mesma unidade da variável dependente); $\mathrm{S}_{\mathrm{xy} \%}^{\text {ajust }}$ $=$ erro padrão da estimava em porcentagem; GLerro = graus de liberdade do erro; ${ }^{*}$ p-valor: $<2,2.10^{-16}$. 
padrão da estimativa. Observa-se ainda similaridade entre os coeficientes dos modelos ajustados para os diferentes tratamentos. Esse resultado é um indicativo da similaridade do comportamento da umidade de equilíbrio dos painéis avaliados em relação às faixas de umidade relativa e temperaturas consideradas, apesar de que em termos médios, em algumas condições de temperatura e umidade relativa do ar, houve influência significativa das diferentes condições de processamento avaliadas,

TABELA 6: Parâmetros e estatísticas referentes ao modelo ajustado 2 para as diferentes condições de processamento. TABLE 6: Parameters and statistics relating to model adjusted 2 for the different processing conditions.

\begin{tabular}{|c|c|c|c|c|c|c|c|c|}
\hline C. de processamento & & icientes & tc & $\mathrm{Fc}$ & $\mathrm{R}_{\text {ajust }}^{2}$ & $S_{x y}$ & $S_{x y \%}$ & GLerro \\
\hline \multirow{6}{*}{1} & $\beta_{0}$ & $-4,518.10^{1}$ & $-7,18^{* *}$ & \multirow{6}{*}{$877,4^{* *}$} & \multirow{6}{*}{0,9684} & \multirow{6}{*}{0,92} & \multirow{6}{*}{8,64} & \multirow{6}{*}{138} \\
\hline & $\beta_{1}$ & $2,539.10^{0}$ & $8,86^{* *}$ & & & & & \\
\hline & $\beta_{2}$ & $-4,335 \cdot 10^{-2}$ & $-9,51^{* *}$ & & & & & \\
\hline & $\beta_{3}$ & $2,559.10^{-4}$ & $10,98^{* *}$ & & & & & \\
\hline & $\beta_{4}$ & $1,337 \cdot 10^{-1}$ & $1,03^{\mathrm{ns}}$ & & & & & \\
\hline & $\beta_{5}$ & $-2,160.10^{-3}$ & $-1,33^{\text {ns }}$ & & & & & \\
\hline \multirow{6}{*}{2} & $\beta_{0}$ & $-4,879.10^{1}$ & $-6,95^{* *}$ & \multirow{6}{*}{$757^{* *}$} & \multirow{6}{*}{0,9640} & \multirow{6}{*}{1,01} & \multirow{6}{*}{9,50} & \multirow{6}{*}{136} \\
\hline & $\beta_{1}$ & $2,659.10^{0}$ & $8,33^{* *}$ & & & & & \\
\hline & $\beta_{2}$ & $-4,526.10^{-2}$ & $-8,91^{* *}$ & & & & & \\
\hline & $\beta_{3}$ & $2,662 \cdot 10^{-4}$ & $10,24^{* *}$ & & & & & \\
\hline & $\beta_{4}$ & $1,847 \cdot 10^{-1}$ & $1,26^{\mathrm{ns}}$ & & & & & \\
\hline & $\beta_{5}$ & $-2,747.10^{-3}$ & $-1,51^{\mathrm{ns}}$ & & & & & \\
\hline \multirow{6}{*}{3} & $\beta_{0}$ & $-4,124.10^{1}$ & $-5,97^{* *}$ & \multirow{6}{*}{$739,2^{* *}$} & \multirow{6}{*}{0,9639} & \multirow{6}{*}{1,00} & \multirow{6}{*}{9,85} & \multirow{6}{*}{137} \\
\hline & $\beta_{1}$ & $2,337 \cdot 10^{0}$ & $7,42^{* *}$ & & & & & \\
\hline & $\beta_{2}$ & $-4,009 \cdot 10^{-2}$ & $-8,01^{* *}$ & & & & & \\
\hline & $\beta_{3}$ & $2,393.10^{-4}$ & $9,34^{* *}$ & & & & & \\
\hline & $\beta_{4}$ & $1,020.10^{-1}$ & $0,71^{\mathrm{ns}}$ & & & & & \\
\hline & $\beta_{5}$ & $-1,663 \cdot 10^{-3}$ & $-0,93^{\mathrm{ns}}$ & & & & & \\
\hline \multirow{6}{*}{4} & $\beta_{0}$ & $-4,228.10^{1}$ & $-6,92^{* *}$ & \multirow{6}{*}{$965,5^{* *}$} & \multirow{6}{*}{0,9712} & \multirow{6}{*}{0,89} & \multirow{6}{*}{8,22} & \multirow{6}{*}{138} \\
\hline & $\beta_{1}$ & $2,371.10^{0}$ & $8,53^{* *}$ & & & & & \\
\hline & $\beta_{2}$ & $-4,079.10^{-2}$ & $-9,23^{* *}$ & & & & & \\
\hline & $\beta_{3}$ & $2,438 \cdot 10^{-4}$ & $10,78^{* *}$ & & & & & \\
\hline & $\beta_{4}$ & $1,535.10^{-1}$ & $1,21^{\mathrm{ns}}$ & & & & & \\
\hline & $\beta_{5}$ & $-2,233 \cdot 10^{-3}$ & $-1,41^{\mathrm{ns}}$ & & & & & \\
\hline \multirow{6}{*}{5} & $\beta_{0}$ & $-4,099.10^{1}$ & $-6,14^{* *}$ & \multirow{6}{*}{$789^{* *}$} & & & & \\
\hline & $\beta_{1}$ & $2,185.10^{0}$ & $7,20^{* *}$ & & & & & \\
\hline & $\beta_{2}$ & $-3,778.10^{-2}$ & $-7,83^{* *}$ & & 00650 & 0.07 & 0 & 120 \\
\hline & $\beta_{3}$ & $2,280.10^{-4}$ & $9,24^{* *}$ & & 0,9050 & 0,91 & $9, \angle 0$ & 158 \\
\hline & $\beta_{4}$ & $2,794.10^{-1}$ & $2,02^{*}$ & & & & & \\
\hline & $\beta_{5}$ & $-3,925.10^{-3}$ & $-2,28^{*}$ & & & & & \\
\hline & $\beta_{0}$ & $-4,234.10^{1}$ & $-7,51^{* *}$ & & & & & \\
\hline & $\beta_{1}$ & $2.379 .10^{0}$ & $9,26^{* *}$ & & & & & \\
\hline 6 & $\beta_{2}$ & $-4.074 .10^{-2}$ & $-9,96^{* *}$ & $1040^{* * *}$ & 00738 & 082 & 706 & 136 \\
\hline 6 & $\beta_{3}$ & $2.419 .10^{-4}$ & $11,55^{* *}$ & 1049 & 0,9138 & 0,82 & 1,96 & 136 \\
\hline & $\beta_{4}$ & $1.422 .10^{-1}$ & $1,20^{\mathrm{ns}}$ & & & & & \\
\hline & $\beta_{5}$ & $-2.299 .10^{-3}$ & $-1,55^{\text {ns }}$ & & & & & \\
\hline
\end{tabular}

Em que: $\mathrm{C}$. de processamento = condições de processamento; $\mathrm{tc}=$ valor de $\mathrm{t}$ calculado; $\mathrm{Fc}=$ valor de $\mathrm{F}$ calculado; $\mathrm{R}^{2}{ }_{\text {ajust }}$ = coeficiente de determinação ajustado; $\mathrm{S}_{\mathrm{xy}}=$ erro padrão da estimativa (mesma unidade da variável dependente); $\mathrm{S}_{\mathrm{xy} \%}$ $=$ erro padrão da estimava em porcentagem; GLerro $=$ graus de liberdade do erro; $* *$ p-valor $=<2,2.10^{-16} ;{ }^{*}$ - -valor $=$ $<0,05 ;$ ns $=$ p-valor $>0,05$. 
conforme discutido anteriormente.

Na Tabela 6 estão apresentados os parâmetros e estatísticas referentes ao modelo ajustado 2 para as diferentes condições de processamento. Observa-se que os modelos não apresentaram os coeficientes $\beta_{4}$ e $\beta_{5}$ significativos pelo Teste $\mathrm{t}$, com exceção ao modelo ajustado para a condição de processamento 5 .

Comparando-se os resultados obtidos para os modelos 1 e 2 pode-se afirmar que houve um efeito linear da temperatura média do ar na umidade de equilíbrio dos painéis OSB avaliados. Isso porque o efeito parabólico da temperatura testado no modelo 2 foi não significativo, enquanto que o efeito linear da temperatura representado pelo coeficiente $\beta_{4}$ do modelo 1 foi significativo pelo teste t a 5\% de significância. Quanto aos erros padrões da estimativa encontrados, observa-se similaridade aos resultados encontrados para modelo 1 dos diferentes painéis avaliados.

TABELA 7: Parâmetros e estatísticas referentes ao modelo ajustado 3 para as diferentes condições de processamento.

TABLE 7: Parameters and statistics relating to model adjusted 3 for the different processing conditions.

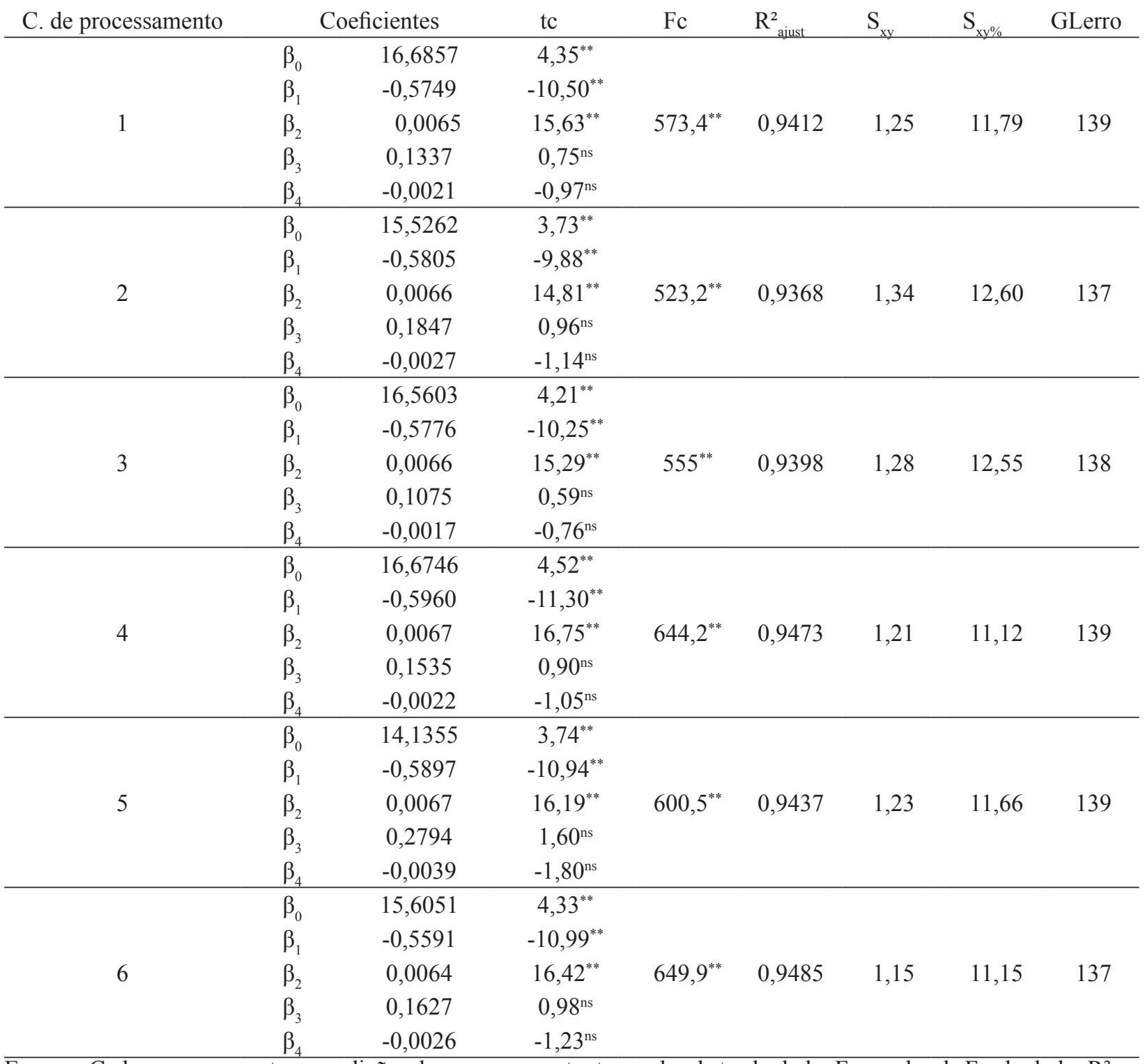

Em que: $\mathrm{C}$. de processamento $=$ condições de processamento; $\mathrm{tc}=$ valor de $\mathrm{t}$ calculado; $\mathrm{Fc}=$ valor de $\mathrm{F}$ calculado; $\mathrm{R}^{2}$ just = coeficiente de determinação ajustado; $\mathrm{S}_{\mathrm{xy}}=$ erro padrão da estimativa (mesma unidade da variável dependente); $\mathrm{S}_{\mathrm{xy} \%}=$ erro padrão da estimava em porcentagem; GLerro $=$ graus de liberdade do erro; ${ }^{* *}$ p-valor $=<2,2.10^{-16}$; ns $=$ p-valor $>0,05$. 
Na Tabela 7 estão apresentados os parâmetros e estatísticas referentes ao modelo ajustado 3 para as diferentes condições de processamento.

Na Tabela 8 estão apresentados os parâmetros e estatísticas referentes ao modelo ajustado 4 para as diferentes condições de processamento.

Apesar de elevado coeficiente de determinação ajustado, os modelos 3 e 4 apresentaram elevado erro padrão da estimativa em relação ao modelo 1 para todos os tratamentos. Esses resultados reforçam a tendência observada nesse trabalho da influência linear da temperatura sobre a umidade de equilíbrio dos painéis, bem como o comportamento polinomial de terceira ordem da umidade de equilíbrio em função apenas da umidade relativa observado no modelo 1 .

Com base nas medidas de precisão e nos resultados obtidos, quando avaliadas as condições de processamento de forma separada, recomendase a utilização do modelo 1 para a estimativa indireta da umidade de equilíbrio dos painéis OSB, considerando-se apenas a umidade relativa e a temperatura média do ar.

$\mathrm{Na}$ Figura 2 encontram-se as curvas de nível para os modelos ajustados dos painéis OSB avaliados. Observa-se comportamento similar da umidade de equilíbrio dos painéis OSB avaliados, sendo o modelo 1 o mais adequado para a determinação das curvas, com exceção do tratamento 5, que apresentou para as umidades relativas de 40

TABELA 8: Parâmetros e estatísticas referentes ao modelo ajustado 4 para as diferentes condições de processamento.

TABLE 8: Parameters and statistics relating to model adjusted 4 for the differentprocessing conditions.

\begin{tabular}{|c|c|c|c|c|c|c|c|c|}
\hline C. de processamento & & Coeficientes & tc & $\mathrm{Fc}$ & $\mathrm{R}_{\text {aijust }}^{2}$ & $\mathrm{~S}_{\mathrm{xy}}$ & $\mathrm{S}_{\mathrm{xy} \%}$ & GLerro \\
\hline \multirow{4}{*}{1} & $\beta_{0}$ & 19,9984 & $11,28^{* *}$ & \multirow{4}{*}{$764,5^{* *}$} & \multirow{4}{*}{0,9412} & \multirow{4}{*}{1,25} & \multirow{4}{*}{11,78} & \multirow{4}{*}{140} \\
\hline & $\beta_{1}$ & $-0,5749$ & $-10,50^{* *}$ & & & & & \\
\hline & $\beta_{2}$ & 0,0065 & $15,64^{* *}$ & & & & & \\
\hline & $\beta_{3}$ & $-0,0391$ & $-3,06^{*}$ & & & & & \\
\hline \multirow{4}{*}{2} & $\beta_{0}$ & 19,7472 & $10,37^{* *}$ & \multirow{4}{*}{$695,6^{* *}$} & \multirow{4}{*}{0,9366} & \multirow{4}{*}{1,34} & \multirow{4}{*}{12,61} & \multirow{4}{*}{138} \\
\hline & $\beta_{1}$ & $-0,5808$ & $-9,88^{* *}$ & & & & & \\
\hline & $\beta_{2}$ & 0,0066 & $14,81^{* *}$ & & & & & \\
\hline & $\beta_{3}$ & $-0,0351$ & $-2,56^{*}$ & & & & & \\
\hline \multirow{4}{*}{3} & $\beta_{0}$ & 19,2006 & $10,57^{* *}$ & \multirow{4}{*}{$742^{* *}$} & \multirow{4}{*}{0,9400} & \multirow{4}{*}{1,28} & \multirow{4}{*}{12,53} & \multirow{4}{*}{139} \\
\hline & $\beta_{1}$ & $-0,5773$ & $-10,26^{* *}$ & & & & & \\
\hline & $\beta_{2}$ & 0,0066 & $15,31^{* *}$ & & & & & \\
\hline & $\beta_{3}$ & $-0,0305$ & $-2,31^{*}$ & & & & & \\
\hline \multirow{4}{*}{4} & $\beta_{0}$ & 20,0982 & $11,77^{* *}$ & \multirow{4}{*}{$857,9^{* *}$} & \multirow{4}{*}{0,9473} & \multirow{4}{*}{1,21} & \multirow{4}{*}{11,12} & \multirow{4}{*}{140} \\
\hline & $\beta_{1}$ & $-0,5960$ & $-11,30^{* *}$ & & & & & \\
\hline & $\beta_{2}$ & 0,0067 & $16,75^{* *}$ & & & & & \\
\hline & $\beta_{3}$ & $-0,0251$ & $-2,04 *$ & & & & & \\
\hline \multirow{4}{*}{5} & $\beta_{0}$ & 20,1538 & $11,46^{* *}$ & \multirow{4}{*}{$786,9^{* *}$} & \multirow{4}{*}{0,9428} & \multirow{4}{*}{1,24} & \multirow{4}{*}{11,76} & \multirow{4}{*}{140} \\
\hline & $\beta_{1}$ & $-0,5897$ & $-10,85^{* *}$ & & & & & \\
\hline & $\beta_{2}$ & 0,0067 & $16,06^{* *}$ & & & & & \\
\hline & $\beta_{3}$ & $-0,0346$ & $-2,73^{* *}$ & & & & & \\
\hline \multirow{4}{*}{6} & $\beta_{0}$ & 19,5663 & $11,88^{* *}$ & \multirow{4}{*}{$862,7^{* *}$} & & & & \\
\hline & $\beta_{1}$ & $-0,5606$ & $-11,00^{* *}$ & & 0.9483 & 1.16 & 1118 & 138 \\
\hline & $\beta_{2}$ & 0,0064 & $16,42^{* *}$ & & & 1,10 & 11,10 & 150 \\
\hline & $\beta_{3}$ & $-0,0417$ & $-3,54^{* *}$ & & & & & \\
\hline
\end{tabular}



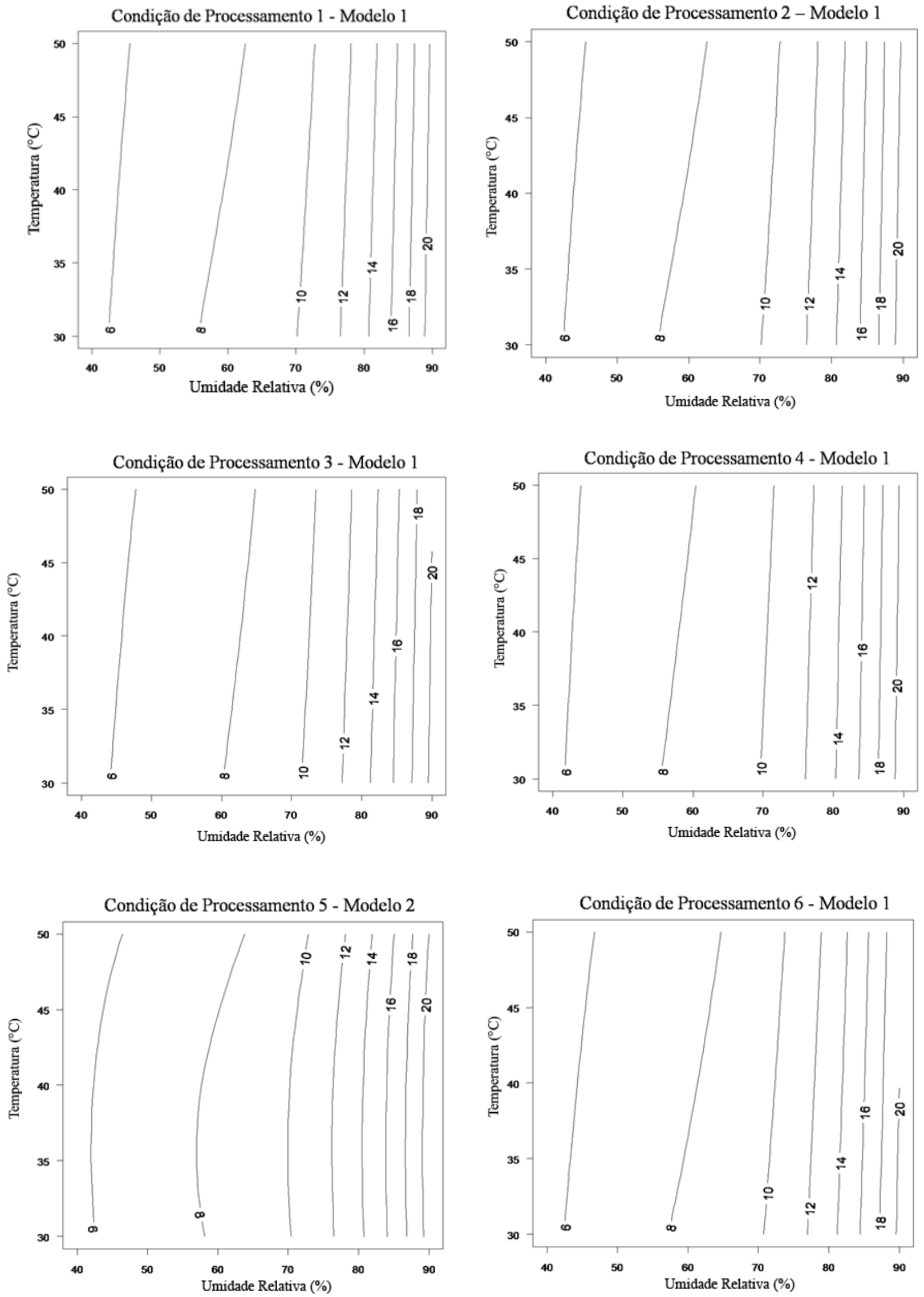

FIGURA 2: Curvas de nível para os modelos ajustados dos painéis OSB avaliados.

FIGURE 2: Contours for the models adjusted of the OSB panels evaluated. 
a $60 \%$, comportamento quadrático da umidade de equilíbrio em decorrência da influência quadrática da temperatura nessa propriedade do painel, sendo o modelo 2 o mais adequado.

A análise gráfica das curvas de nível evidencia maior influência da umidade relativa do que da temperatura na umidade de equilíbrio dos painéis OSB, o mesmo foi referenciado por Siau (1995) para a madeira maciça. Pode-se observar ainda que à medida que se aumenta a umidade relativa do ar, as curvas de nível ficam mais próximas e menos inclinadas, aumentando assim o efeito direto da umidade relativa do ar em relação à umidade de equilíbrio dos painéis OSB.

Verifica-se pelas curvas de nível que, assim como observado pelo teste de comparação múltipla aplicado (Tabela 4), os painéis produzidos com as partículas strand com a espessura de 1,0 $\mathrm{mm}$ (Condição de processamento 3 ) apresentaram tendência de diminuir a umidade de equilíbrio dos painéis OSB, visto que as curvas de umidade de equilíbrio dos painéis encontram-se deslocadas no sentido das maiores umidades relativas do ar.

Ainda em relação às curvas de nível, não foi possível diferenciar o efeito da densidade aparente $\left(0,65\right.$ e $\left.0,90 \mathrm{~g} / \mathrm{cm}^{3}\right)$ sobre a umidade de equilíbrio dos painéis OSB, assim como foi observado em termos médios na análise estatística realizada anteriormente (Tabela 4). Esse resultado é um indicativo da viabilidade da utilização de modelos gerais considerando todas as condições de processamento para a estimativa indireta da umidade de equilíbrio dos painéis OSB (Tabela 9).

Contudo, as curvas de nível se mostram com grande praticidade de utilização, apesar de não ser possível perceber diferenças entre as variáveis de produção, certamente em função de pequenas aproximações de valores promovida pela utilização dos modelos. Enquanto que para as diferentes pressões de prensagem, observa-se que é o fator

TABELA 9: Modelos gerais ajustados considerando todas as condições de processamento para a estimativa da umidade de equilíbrio dos painéis OSB e estatísticas utilizadas na avaliação dos ajustes.

TABLE 9: General models adjusted considering all the processing conditions to estimate the equilibrium moisture content of OSB panels and statistics used to assess the adjustments.

\begin{tabular}{|c|c|c|c|c|c|c|c|c|}
\hline Modelos & & ficientes & tc & $\mathrm{Fc}$ & $\mathrm{R}^{2}$ & $\mathrm{~S}_{\mathrm{xu}}$ & $\mathrm{S}_{\mathrm{xy} \%}$ & GLerro \\
\hline \multirow{5}{*}{1} & $\beta_{0}$ & $-3,965.10^{1}$ & $-16,041^{* *}$ & \multirow{5}{*}{$6088^{* *}$} & \multirow{5}{*}{0,9660} & \multirow{5}{*}{0,96} & \multirow{5}{*}{9,10} & \multirow{5}{*}{854} \\
\hline & $\beta_{1}$ & $2,413.10^{0}$ & $19,700^{* *}$ & & & & & \\
\hline & $\beta_{2}$ & $-4,135 \cdot 10^{-2}$ & $-21,230^{* *}$ & & & & & \\
\hline & $\beta_{3}$ & $2,459 \cdot 10^{-4}$ & $24,674^{* *}$ & & & & & \\
\hline & $\beta_{4}$ & $-3,454.10^{-2}$ & $-8,626^{* *}$ & & & & & \\
\hline \multirow{6}{*}{2} & $\beta_{0}$ & $4,348.10^{1}$ & $-16,272^{* *}$ & \multirow{6}{*}{$4943^{* *}$} & \multirow{6}{*}{0,9664} & \multirow{6}{*}{0,95} & \multirow{6}{*}{9,04} & \multirow{6}{*}{853} \\
\hline & $\beta_{1}$ & $2,412.10^{0}$ & $19,830^{* *}$ & & & & & \\
\hline & $\beta_{2}$ & $-4,133.10^{-2}$ & $-21,370^{* *}$ & & & & & \\
\hline & $\beta_{3}$ & $2,458 \cdot 10^{-4}$ & $24,837^{* *}$ & & & & & \\
\hline & $\beta_{4}$ & $1,658 \cdot 10^{-1}$ & $2,990^{*}$ & & & & & \\
\hline & $\beta_{5}$ & $-2,504 \cdot 10^{-3}$ & $-3,622^{* *}$ & & & & & \\
\hline \multirow{5}{*}{3} & $\beta_{0}$ & 15,8577 & $10,101^{* *}$ & \multirow{5}{*}{$3500^{* *}$} & \multirow{5}{*}{0,9422} & \multirow{5}{*}{1,25} & \multirow{5}{*}{11,85} & \multirow{5}{*}{854} \\
\hline & $\beta_{1}$ & $-0,5792$ & $-25,902^{* *}$ & & & & & \\
\hline & $\beta_{2}$ & 0,0066 & $38,591^{* *}$ & & & & & \\
\hline & $\beta_{3}$ & 0,1701 & $2,337^{*}$ & & & & & \\
\hline & $\beta_{4}$ & $-0,0026$ & $-2,818^{*}$ & & & & & \\
\hline \multirow{4}{*}{4} & $\beta_{0}$ & 19,7843 & $27,243^{* *}$ & \multirow{4}{*}{$4626^{* *}$} & \multirow{4}{*}{0,9418} & \multirow{4}{*}{1,26} & \multirow{4}{*}{11,90} & \multirow{4}{*}{855} \\
\hline & $\beta_{1}$ & $-0,5795$ & $-25,808^{* *}$ & & & & & \\
\hline & $\beta_{2}$ & 0,0066 & $38,446^{* *}$ & & & & & \\
\hline & $\beta_{3}$ & $-0,0344$ & $-6,575^{* *}$ & & & & & \\
\hline
\end{tabular}

Em que: $\mathrm{Tc}=$ valor de $\mathrm{t}$ calculado; $\mathrm{Fc}=$ valor de $\mathrm{F}$ calculado; $\mathrm{R}_{\text {ajust }}^{2}=$ coeficiente de determinação ajustado; $\mathrm{S}_{\mathrm{xy}}=$ erro padrão da estimativa (mesma unidade da variável dependente); $\mathrm{S}_{\mathrm{xy} \%}=$ erro padrão da estimava em porcentagem; GLerro $=$ graus de liberdade do erro; $* *=p$-valor $<2,2.10^{-16} ; *=p$-valor $<0,05$. 

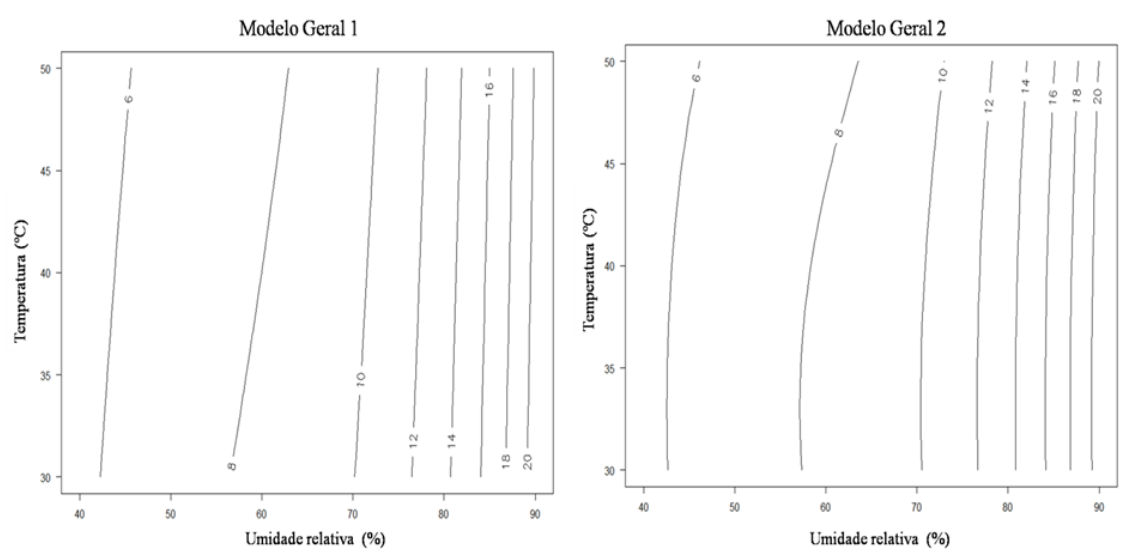

FIGURA 3: Curvas de nível referentes aos modelos gerais ajustados 1 e 2.

FIGURE 3: Contour referring to general models adjusted 1 and 2.

que mais promoveu a variação da umidade de equilíbrio dos painéis OSB. Podendo perceber certa diminuição da umidade de equilíbrio dos painéis OSB quando esses foram prensados na pressão de $80 \mathrm{kgf} / \mathrm{cm}^{2}$, assim como verificado no teste de comparação múltipla de médias (Tabela 4).

Observa-se que dentre os modelos ajustados considerando todas as condições de processamento (Tabela 9), os modelos 1 e 2 apresentaram os melhores resultados, se destacando pelo alto coeficiente de determinação ajustado e baixo erro padrão da estimativa. Esse resultado é um indicativo da viabilidade do uso de modelos gerais para a estimativa da umidade de equilíbrio dos painéis OSB com base apenas na umidade relativa do ar e da temperatura ambiente e da baixa influência das condições de processamento estudadas nessa variável dependente. A representação das curvas de nível dos modelos gerais 1 e 2 ajustados encontrase na Figura 3. Observou-se que os valores preditos assemelharam-se aos observados. Portanto, a escala de predição é tecnicamente viável, justificando o baixo erro padrão da estimativa (Tabela 9).

De forma geral, o modelo 1 foi o que apresentou os melhores ajustes para a maioria das condições de processamento, quando elas foram avaliadas de forma separada, e foi um dos dois melhores modelos para a avaliação geral da umidade de equilíbrio de painéis OSB. Além disso, é um modelo relativamente mais simples que o modelo 2 , destacando-se assim como o melhor modelo para a determinação da umidade de equilíbrio dos painéis OSB com base na umidade relativa do ar e da temperatura ambiente.

\section{CONCLUSÕES}

Com base nas medidas de precisão e nos resultados obtidos recomenda-se a utilização do modelo 1 (UEQ $=\beta_{0}+\beta_{1} \mathrm{UR}+\beta_{2} \mathrm{UR}^{2}+\beta_{3} \mathrm{UR}^{3}+$ $\beta_{4}$ Temp $+\varepsilon$ ) para a estimativa indireta da umidade de equilíbrio dos painéis OSB.

A temperatura apresenta influência linear na umidade de equilíbrio dos painéis, enquanto que a umidade relativa do ar apresenta comportamento polinomial de terceira ordem. Sendo que a umidade relativa do ar influencia de forma mais pronunciada a umidade de equilíbrio dos painéis OSB do que a temperatura ambiente.

Quanto ao efeito das variáveis de produção, a pressão de prensagem de $80 \mathrm{kgf} / \mathrm{cm}^{2}$ e o aumento da espessura das partículas strand para $1,0 \mathrm{~mm}$ de espessura promoveu tendência de reduções nos valores médios de umidade de equilíbrio dos painéis OSB. Enquanto que o aumento da densidade do painel promoveu a tendência do aumento dos valores médios de umidade de equilíbrio dos painéis OSB.

O uso de modelos estatísticos lineares múltiplos permite que sejam produzidas curvas de nível para a obtenção dos valores de umidade de equilíbrio dos painéis OSB em função da umidade relativa e da temperatura do local em que o painel será utilizado, destacando-se pela sua praticidade de utilização. Contudo, não é possível perceber influências geradas pelas variáveis de produção.

\section{REFERÊNCIAS BIBLIOGRÁFICAS}

BAHAROGLU, $M$. et al. The influence of 
moisture content of raw material on the physical and mechanical properties, surface roughness, wettability and formaldehyde emission of particleboard composite. Composites: Part B. v. 43, pg. 2448-2451, 2012.

HASELEIN, C.R. et al. Resistência mecânica e à umidade de painéis aglomerados com partículas de Madeira de diferentes dimensões. Ciência Florestal, Santa Maria, v. 12, n. 2, pg. 127-134, 2002.

MARTINS, V.A. et al. Umidade de equilíbrio e risco de apodrecimento da madeira em condições de serviço no Brasil. Brasil Florestal, Brasília, n.76, p.29-34, 2003.

MENDES, L. M. ; MENDES, R. F. ; PEIXOTO, M.G.M. . Equilibrium moisture content of wood panels. In: International union of forest research organizations - IUFRO, 2012, Lisboa - Portugal. IUFRO 2012. LISBOA: IUFRO, v. 1, pg. 228-228, 2012 b.

MENDES, L.M.; ARCE, J.E.. Análise comparativa das equações utilizadas para estimar a umidade de equilíbrio da madeira. Cerne, Lavras, v.9, n. 2, p. 141-152, jul./dez. 2003.

MENDES, L.M. et al. Influência do teor de resina, temperatura e tempo de prensagem na umidade de equilíbrio de painéis de partículas de madeira. Cerne, Lavras, v. 12, n. 4, p. 329-335, out./dez. 2006.
MENDES, R.F. et al. Effect of thermal treatment on properties of OSB panels. Wood Science and Technology. First online, pg. 494 - 497, 2012a.

NELSON, R. M. A model for sorption of water vapor by cellulosic materials. Wood Fiber Science, Madison, v. 15, n. 1, p. 8-22, 1983.

SIAU, J.F. Wood: influence of moisture on physical properties. Blacksburg: Virginia Polytechnic Institute and State University. Department of Wood Science and Forest Products, 227p, 1995.

SILVA, G. A. et al. Estimativa da umidade de equilíbrio de painéis de madeira. Scientia Forestalis, Piracicaba, n. 70, p 23-29, 2006.

SILVA, G. A. et al. Umidade de equilíbrio de painéis de madeira. Revista Árvore, Viçosa-MG, v.29, n.4, p.639-646, 2005.

SIMPSON, W.T.; TENWOLDE, A. Physical properties and moisture relations of wood. In: USDA FOREST SERVICE. Wood handbook: wood as an engineering material. Madison: Forest Products Laboratory, Cap.3, 1999.

WERKEMA, C.; AGUIAR, S. Análise de regressão: como entender 0 relacionamento das variáveis de um processo. Belo Horizonte: Werkema, p. 306, 2006.

WU, Q. Apllication of Nelson's sorption isotherm to wood composites and overlays. Wood Fiber Science, Madison, v. 3, n. 2, 187-191, 1999. 